\title{
Temperatures and Optical Depths of Saturn's Rings and a Brightness Temperature for Titan
}

\author{
LuCIEN FroideVAuX AND ANDRew P. INGERSOLl
}

\author{
Division of Geological and Planetary Sciences, California Institute of Technology
}

Pasadena, California 91125

\begin{abstract}
The Pioneer Saturn infrared radiometer viewed Saturn's rings at 20- and 45- $\mu \mathrm{m}$ wavelength under several conditions of illumination. The data are analyzed to infer radial locations of major ring boundaries, temperatures and temperature gradients, and normal optical depths. Error bounds on the above inferred quantities are given. Most ring boundaries are defined to $\pm 0.01 R_{S}\left(1 R_{S}=6 \times 10^{4} \mathrm{~km}\right)$ and are in good agreement with those inferred from the imaging photopolarimeter experiment. Temperatures generally decrease with radial distance from the planet. A significant temperature gradient exists from the colder north (unilluminated) side of the rings to the warmer south side. The gradient appears to be steepest on the south side. Ring optical depths are greater than some previously published values and are approximately 0.1 for the Cassini division and the $\mathrm{C}$ ring. In addition, the $\mathrm{C}$ ring optical depth decreases towards the planet. The temperature drop during eclipse is $\geqq 10 \mathrm{~K}$, implying low thermal inertia for the ring particles. Titan's $45-\mu \mathrm{m}$ brightness temperature is $75 \pm 5 \mathrm{~K}$, in good agreement with earth-based observations.
\end{abstract}

\section{INTRODUCTION}

The Pioneer Saturn infrared radiometer (IRR) viewed Saturn's rings against space and against the planetary disk, at instrument elevation angles with respect to the ring plane ranging from $0^{\circ}$ to $30^{\circ}$ on the south (illuminated) side and from $0^{\circ}$ to $1^{\circ}$ on the north (unilluminated) side. A small number of observations were made of the south side in Saturn's shadow. Ring temperatures were too low to produce significant emission in the 20- $\mu \mathrm{m}$ channel at the time of the Pioneer flyby, owing to the low value $\left(2.83^{\circ}\right)$ of the solar elevation angle with respect to the ring plane. A 45- $\mu \mathrm{m}$ color image and an accompanying drawing of the viewing geometry are shown in a preliminary report [Ingersoll et al., 1980]. Characteristics of the filters and an analysis of Saturn planet data are given by $O r$ ton and Ingersoll [this issue].

The boundaries of the $A$ ring, the Cassini division, the outer and inner parts of the $B$ ring, and the $C$ ring are all defined in the infrared image. The preliminary report gave radial locations of these boundaries, temperatures of the north and south sides, and normal optical depths for each ring. Below we present a more thorough discussion of the determination of these parameter values and their uncertainties.

The next section deals with the location of ring boundaries. Most of the uncertainty arises from the finite resolution of the instrument. Spacecraft trajectory and pointing uncertainties contribute to a lesser extent. We then discuss the north side temperature. The derived value is significantly lower than the south (illuminated) side temperature. Titan is discussed at this point because the data analysis is similar to that for the north side of the rings. We then present a simple thermal model of ring temperature and emission as a function of elevation angle. This model is used in a least squares fit to the data. Subsequent sections give the derived model parameters for the $A$ ring, the Cassini division, the $B$ ring, and the $C$ ring. The $\mathrm{C}$ ring section also includes a discussion of temperatures in the planet's shadow. A brief discussion mentions previous observations and lists a number of answered and unanswered questions.

Copyright (C) 1980 by the American Geophysical Union.

\section{RING BOUNDARIES}

Figure 1 shows a curve of average $45-\mu \mathrm{m}$ brightness versus radial distance $r$ from the center of Saturn in units of Saturn radii $R_{S}\left(1 R_{S} \equiv 6 \times 10^{4} \mathrm{~km}\right)$. For $r \leq 2.13 R_{S}$, only data for which the rings were viewed against Saturn are included. For $r>2.13 R_{s}$, no such data were obtained, so the data shown are for rings viewed against space. The curves were made by separating the data into bins $0.01 R_{S}$ wide and averaging the raw data numbers (DN) in each bin to produce a single brightness for each radial location. The viewing angles included in this averaging are all larger than $11^{\circ}$. This lower limit was set in order to gain finer resolution in the location of the ring boundaries and, in particular, the narrow divisions. This procedure ignores the dependence of brightness on elevation angle. Thus the quantitative brightness amplitudes in Figure 1 are of little significance. The important qualitative feature is that optically thin portions of the rings permit more intense planetary emission to shine through (for $r \leq 2.13 R_{S}$ ).

The radial locations of ring boundaries inferred from Figure 1 are summarized in Table 1 . Since the instrument field of view is $0.3^{\circ}$ in the direction of spacecraft motion and $1.0^{\circ}$ in the perpendicular direction (along the scan and word axes, respectively, in Figure 1 of Ingersoll et al. [1980]) and since the rings were viewed at distances of $1.0 R_{S}$ or greater, the ring boundaries are uncertain to $\pm 0.01 R_{s}$ due to resolution. The uncertainties due to instrument pointing with respect to the spacecraft, spacecraft attitude, and spacecraft trajectory are more difficult to assess. A conservative estimate is obtained by comparing several versions of the trajectory analysis with the final analysis. The changes in $r$ for individual observations are less than $0.01 R_{S}$ and are therefore neglected.

It is not clear, from those infrared observations, where the inner $C$ ring boundary lies, and in Table 1 we have used the value obtained by the imaging photopolarimeter team [Gehrels et al., 1980]. It is plausible that the feature at about 1.52 $R_{S}$ corresponds to the French division, noted by the above team, but our detection of it is only marginal. The feature at about $1.46 R_{s}$ was called 'C ring division' by Ingersoll $\mathrm{et}$ al. [1980], and it appears as a region of lower optical depth than the surroundings. Such a feature was not seen by the photopolarimeter [Gehrels et al., 1980]. We have considered the pos- 


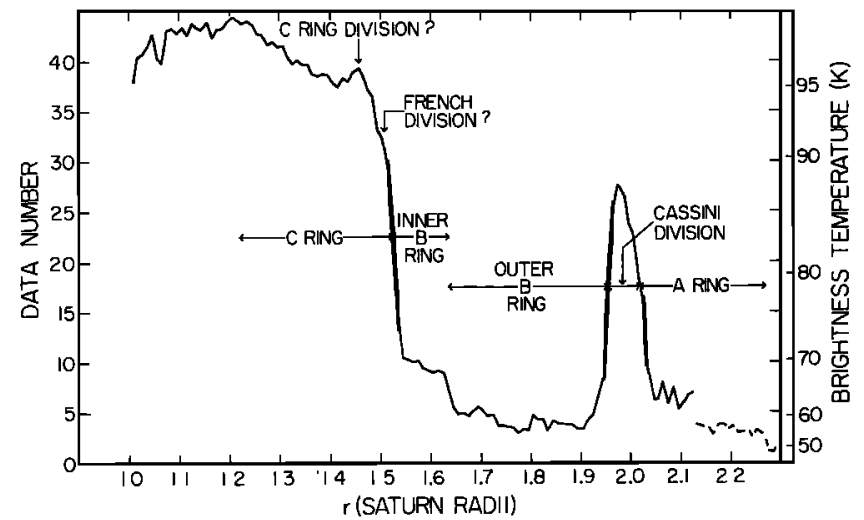

Fig. 1. Brightness of Saturn's rings over the planetary disk at 45 $( \pm 11) \mu \mathrm{m}$. The data have been averaged over elevation angle. Sharp ring boundaries are clearly observed (see Table 1). The inner $C$ ring boundary is taken at $1.22 R_{S}$, following the results of Gehrels et al. [1980]. The dashed curve beyond $2.13 R_{S}$ represents data from the $A$ ring over space, which yield a value for the outer edge of this ring, beyond which the brightness drops to about $1.25 \mathrm{DN}$, corresponding to the noise level over space alone.

sibility that the IRR instrument 'overshoots' in scanning from a cold region (the $B$ ring) to a warm region (the $C$ ring). Anomalies of this sort were observed at Jupiter for the Pioneer 10 instrument but not for Pioneer 11. However, the feature, which is faintly visible in Figure 1 of Ingersoll et al. [1980], is parallel to other ring boundaries, as opposed to being displaced downward from the cold to hot boundary by a constant number of words. Therefore we believe that the feature is a real division in the $C$ ring.

The $B$ ring has been separated into two main components, the outer one being almost 3 times wider than the inner one. The outer edge of the A ring cannot be seen in the data over the planet, since the geometry did not allow such measurements, but we have determined the position of this boundary from the ring data over space (dashed line in Figure 1). The transition occurs at $2.270 R_{s}$, where the brightness drops to 1.25 DN, the value for empty space. The various ring boundaries agree very well with the results of the photopolarimeter observations. There is a small difference between the IRR and photopolarimeter value for the outer edge of the $A$ ring and the value deduced from charged particle experiments. This difference could reflect an ofiset of the magnetic center from the geometric center of the planet [Van Allen et al., 1980].

\section{UNILluminated Side OF THE RINGS, TITAN}

In our preliminary report we stated that the temperature of the north (unilluminated) side of the rings at the time of the Pioneer encounter was $55 \mathrm{~K}$. The small signal to noise ratio and the extreme nonlinearity of the Planck function at $45 \mu \mathrm{m}$ placed an upper limit of $60 \mathrm{~K}$ and a lower limit of zero on this preliminary determination. We have refined the estimate and now report

$$
T \text { (north side) }=54 \pm 3 \mathrm{~K}
$$

The above brightness temperature is derived by averaging 46 individual observations at the same position (word number 18 ) in the spacecraft roll cycle. The rings are viewed at instrument elevation angles of $1^{\circ}$ or less. About $70 \%$ of these observations are of the $A$ ring, $15 \%$ of the Cassini division, and $15 \%$ of the B ring. Factors that were taken into account in the analysis include the small angular size (less than the in- strument field of view) of the rings, the discontinuous word shifts that cause the field of view to walk back and forth across the ring, and the inability of the instrument to sense below $\mathrm{DN}=1$ even though zero intensity corresponds to $\mathrm{DN} \approx$ 0 . These factors were described by Ingersoll et al. [1980] but have been modeled more completely in the present analysis. There is little doubt that the rings were detected. The average data number for the 46 observations is exceeded in comparable observations of space less than $1 \%$ of the time.

The Pioneer IRR observations of the unilluminated side of the rings were followed by earth-based measurements [Tokunaga et al., 1980] in which a $20-\mu \mathrm{m}$ brightness temperature of $56 \pm 1 \mathrm{~K}$ was derived. The smaller error limits are presumably due to the cooler detectors and longer integration times on earth than on the spinning Pioneer spacecraft. The earthbased observations include more of the $B$ ring than ours, and the agreement is good.

Titan presents a similar problem for the IRR instrument: a small, cold object that did not fill the field of view, for which about 30 observations were obtained. In the preliminary report we quoted a disk-averaged $45-\mu \mathrm{m}$ brightness temperature of $80 \pm 10 \mathrm{~K}$. The refined value is now

$$
T(\text { Titan) }=75 \pm 5 \mathrm{~K}
$$

The above value is in excellent agreement with a recently quoted observation of $74 \pm 3.3 \mathrm{~K}$ at $65 \mu \mathrm{m}$ [Loewenstein et al., 1980], taken from the NASA Kuiper airborne observatory. Since Titan radiates more intensely at shorter wavelengths, there is no inconsistency between the relatively cold 45 - and $65-\mu \mathrm{m}$ temperatures and the relatively warmer effective temperature of an object of Titan's albedo in equilibrium with solar radiation at Titan's distance from the sun [Loewenstein et $a l ., 1980]$. The error quoted in (2) is largely due to uncertainty in the location of Titan in the instrument field of view. Instrument noise and calibration uncertainty also contribute. Uncertainty due to Titan's radius $(2800 \pm 100 \mathrm{~km})$ is small.

\section{THERMAL MODEL OF THE RINGS}

For each of the major rings defined in Table 1 and Figure 1 an attempt was made to fit the data down to the noise of a single observation. This required from two to four free parameters. The south side temperature is most sensitive to observations at low instrument elevation angles. The normal optical depth is sensitive to variations of brightness with elevation angle. Gradients of temperature normal to the ring plane are also sensitive to the emission angle variations. This choice of parameters, based on an assumed homogeneous, isotropic, plane-parallel emitting layer, reflects a desire for simplicity rather than a preference for one theory over another.

TABLE 1. Ring Boundaries Obtained from Figure 1

\begin{tabular}{lc}
\hline & Radius, $6 \times 10^{4} \mathrm{~km}$ \\
\hline A ring & $2.020-2.270$ \\
Cassini division & $1.955-2.020$ \\
B ring (outer) & $1.635-1.955$ \\
B ring (inner) & $1.525-1.635$ \\
French division & $1.500-1.525$ \\
C ring division & $1.44-1.48$ \\
C ring & $1.22 *-1.525$
\end{tabular}

The French division was observed by the photopolarimeter [Gehrels et al., 1980], but that instrument did not detect what we refer to as the C ring division.

*From Gehrels et al. [1980]. 
TABLE 2. Model Parameter Estimates for the Various Regions in Saturn's Rings

\begin{tabular}{|c|c|c|c|c|c|c|}
\hline & \multicolumn{2}{|c|}{ A Ring } & \multirow{2}{*}{$\begin{array}{c}\text { Cassini } \\
\text { Division }\end{array}$} & \multicolumn{2}{|c|}{ B Ring } & \multirow[b]{2}{*}{ C Ring } \\
\hline & Average & Inner & & Outer & Inner & \\
\hline $\begin{array}{l}\text { Radial range used, } R_{S} \\
\text { Normal optical depth } \tau_{0} \\
\text { Illuminated side temperature } T_{h}, \mathrm{~K} \\
\text { Gradient parameter } \alpha\end{array}$ & $\begin{array}{l}2.05-2.25 \\
0.58_{-0.08}^{+0.10} \\
64_{-4}^{+7} \\
6 \pm-14\end{array}$ & $\begin{array}{l}2.05-2.10^{*} \\
0.65_{-0.07}^{+0.11} \\
71_{4.5}^{+7.5} \\
9 \pm 11\end{array}$ & $\begin{array}{l}1.96-2.00 \\
0.13+0.03 \\
71 \pm 5 \\
\text { indefinite }\end{array}$ & $\begin{array}{l}1.65-1.90 \\
1.8_{-0.31}^{+\infty} \\
68_{-5}^{+3} \\
8 \pm-5\end{array}$ & $\begin{array}{l}1.55-1.62 \\
0.95 \pm 0.20 \\
70_{-3}^{+5} \\
3_{-3}^{+5}\end{array}$ & $\begin{array}{l}1.25-1.45 \\
0.12 \pm 0.02 \\
86 \pm 2 \\
\text { indefinite }\end{array}$ \\
\hline
\end{tabular}

To avoid contamination in the field of view by neighboring rings or space, the radial ranges used in the data analysis are slightly smaller than the values in Table 1 . The bounds on the parameters listed above are strictly valid for $T_{U}$ (A ring) $\leq 58 \mathrm{~K}$ and $T_{U}$ (B ring) $\leq 56 \mathrm{~K}$.

*Data from A ring over planet only cover the radial range $2.05-2.10 R_{S}$ (see text).

$\dagger$ The outer B ring data can be fit by very high $\tau_{0}$ values (up to $\tau_{0} \simeq 3$ ), but such high optical depths are in strong contradiction with all earthbased results.

$\ddagger$ The $C$ ring and Cassini division residuals are not sensitive to $\alpha$.

The observed brightness from a ring of normal optical depth $\tau_{0}$ is

$$
I(\mu)=\int_{0}^{\tau_{0}} B(\tau) e^{-\tau / \mu} d(\tau / \mu)+B_{p} e^{-\tau_{0} / \mu}
$$

where $\mu$ is the sine of the elevation angle with respect to the ring plane, $B(\tau)$ is the intrinsic ring brightness at optical depth $\tau$, and $B_{P}$ is the planetary brightness behind the ring. If $B(\tau)$ is a constant $B_{0}$, we have

$$
I(\mu)=B_{0}\left(1-e^{-\tau_{0} / \mu}\right)+B_{p} e^{-\tau_{0} / \mu}
$$

Unknown free parameters are now $B_{0}$ and $\tau_{0}$.

However, we will find it useful to introduce a possible gradient of temperature within the rings. In particular, an exponentially decreasing function of the following form is used:

$$
B(\tau)=B_{1} e^{-\alpha \tau}+B_{2}
$$

Denoting the illuminated side (intrinsic) brightness by $B_{I}$ and the unilluminated side brightness by $B_{U}$, we have

$$
\begin{aligned}
& B_{I}=B(0)=B_{1}+B_{2} \\
& B_{U}=B\left(\tau_{0}\right)=B_{1} e^{-\alpha \tau_{0}}+B_{2}
\end{aligned}
$$

Equations (3) and (5) yield

$$
\begin{aligned}
I(\mu)=B_{1}\left[1-e^{-(1+\alpha \mu) r_{0} / \mu}\right](1 & +\alpha \mu)^{-1} \\
& +B_{2}\left(1-e^{-\tau_{0} / \mu}\right)+B_{p} e^{-r_{0} / \mu}
\end{aligned}
$$

Unknown free parameters are $B_{1}, B_{2}, \alpha$, and $\tau_{0}$, although generally $B_{2}$ is not well determined. The number of free parameters may be reduced to three by choosing a value for $B_{2}$ or for the unilluminated side brightness $B_{U}$.

The planetary contribution $B_{P}$ behind the rings depends on latitude and emission angle with respect to the local vertical. We use the Legendre polynomial fit [Orton and Ingersoll, this issue] to southern hemisphere data in order to obtain estimates of $B_{P}$ in the northern hemisphere behind the rings.

Our basic approach is to vary the unknown free parameters $a_{j}$ until the minimum rms residual $\epsilon\left(a_{1}, a_{2}, \cdots, a_{j}\right)=\epsilon_{\min }$ is found. A residual is defined as the difference between an observed $45-\mu \mathrm{m}$ intensity and that computed from a model such as (4) or (7). The model is applied separately to each of the major regions defined in Table 1.

The values of the parameters for $\epsilon=\epsilon_{\min }$ are the least squares estimates $\bar{a}_{j}$. The curvature of the surface $\epsilon\left(a_{1}, a_{2}, \cdots\right.$, $\left.a_{j}\right)$ near the minimum point is used to estimate uncertainties. Equivalently, contours $\epsilon=$ const around $\epsilon_{\min }$ give the $1 \sigma, 2 \sigma$, etc., limits within which the true values $a_{j}$ should lie. For instance, the $1 \sigma$ limit is defined by the contour

$$
\epsilon\left(a_{1}, a_{2}, \cdots, a_{j}\right)=\epsilon_{\min }\left[1+\frac{1}{2(N-m)}\right]
$$

Here $N$ is the number of independent observations, and $m$ is the number of free parameters. This expression may be derived from, for example, Bevington [1969, p. 245]:

$$
\sigma\left(a_{j}\right)=\left[\left(\frac{N-m}{\epsilon}\right) \mid\left(\frac{\partial^{2} \epsilon}{\partial a_{j}^{2}}\right)\right]^{-1 / 2}
$$

which is valid for independent, that is, uncorrelated parameters $a_{p}$ for which

$$
\epsilon=\epsilon_{\min }\left[1+\sum_{j} c_{(}\left(a,-\bar{a}_{j}\right)^{2}\right]
$$

Since independent parameters can be constructed by a linear transformation from the original parameters, the result (8) applies in general. This result may also be derived as the limit of an $F$ test [Bevington, 1969, p. 200] as $N-m \rightarrow \infty$. One takes $F=1$, corresponding to a probability of about two thirds that a random fluctuation will have a smaller $\epsilon$ than the contoured value. Application of (10-10) of Bevington [1969] then leads to the result (8).

For $N$ we have (conservatively) used the number of roll motions of the instrument, as opposed to the total number of data points, since observations from the same roll are referenced to the same observation of space. This typically reduces the number of independent points by a factor of 3 .

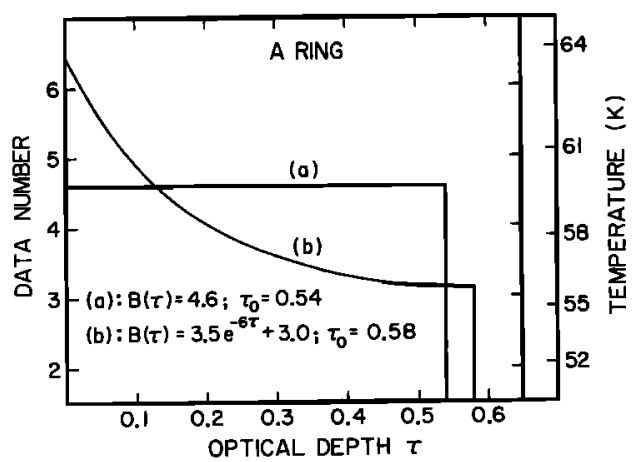

Fig. 2. The intrinsic brightness $B(\tau)$ of the $A$ ring for two models. Model $a$ is the best model with zero gradient, and model $b$ is the best model with nonzero gradient, for $A$ ring over space combined with $A$ ring over planet. 


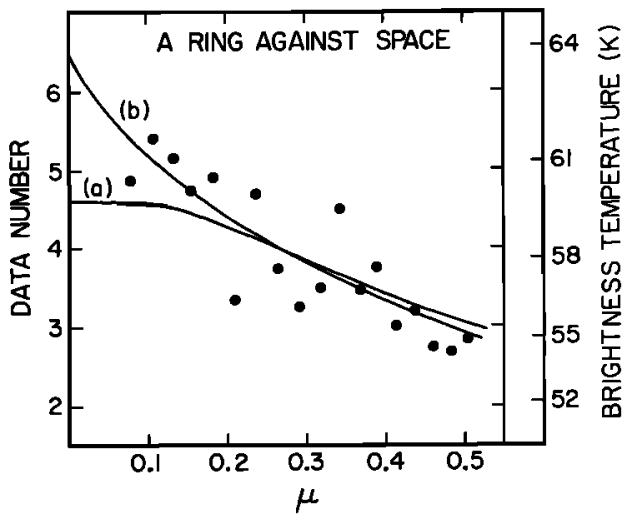

Fig. 3. Fit of the brightness models of Figure 2 to the A ring data over space. Each data point is an average of many soundings at roughly similar elevation angles; the bins are as wide as the field of view, that is, less than $2^{\circ}$. The brightness goes up fairly rapidly as $\mu$ decreases, and a temperature gradient (model $b$ ) within the A ring is indicated.

\section{A RING}

Most of the views of the A ring are with space as a background. About 220 observations covering the range $2.05 \leq r /$ $R_{S} \leq \mathbf{2 . 2 5}$ were selected for analysis. In addition, 11 observations were selected with the planet as a background. These cover only the inner part of the A ring $2.05 \leq r / R_{S} \leq 2.10$. Both data sets are needed to resolve ambiguities concerning the values of $B_{1}, \tau_{0}$, and $\alpha$ in (7).

Data with A ring over space do not provide a useful upper bound on $\tau_{0}$. This is because the temperature gradient $(\alpha>0)$ can closely model the effect of finite (not infinite) $\tau_{0}$, which is to cause brightness to decrease as $\mu$ increases. Data over planet help to resolve this ambiguity, since the effect of finite $\tau_{0}$ is reversed. That is, finite $\tau_{0}$ allows more planetary emission through the rings at larger values of $\mu$.

If the two data sets are combined for the same radial range $\left(2.05-2.10 R_{S}\right)$, one obtains $\tau_{0}=0.65_{-0.07}^{+0.11}$ and $T_{I}=71_{-45}^{+7} \mathrm{~K}$. This holds for values of $T_{U}$ below $58 \mathrm{~K}$, above which the rms residual becomes too large (more than $1 \sigma$ away from the minimum). Therefore a nonzero temperature gradient is clearly preferred (see Table 2). This parameter $\alpha$ is highly correlated with $T_{l}$, in the sense that an acceptable fit to the data can be obtained for very high values of $\alpha$, if $T_{I}$ is increased as well. Since this is more a mathematical artifact than a realistic picture of the temperature gradient within the ring, we have chosen to limit $\alpha$ to values below 20. In most cases (for the $A$ and $B$ rings) a value of $\alpha>20$ yields an rms residual outside the $1 \sigma$ limit.

We also obtained parameter estimates when the whole radial range (2.05-2.25 $R_{S}$ ) was allowed. The temperature decreases to $T_{I}=64_{-4}^{+7} \mathrm{~K}$, while the optical depth is lowered to $\tau_{0}$ $=0.58_{-0.08}^{+0.10}$. A shallower (but still nonzero) temperature gradient is indicated (see Table 2). The latter parameter values are close to the average $A$ ring values, since the data span the entire A ring. However, the data for ring over planet (see last paragraph) do not go beyond $2.10 \boldsymbol{R}_{S}$, and it could be that the estimates of $\tau_{0}$ and $T_{I}$ would be slightly lowered if the ring had been observed (against Saturn) at larger radial distances. We conclude from this analysis that both the optical depth and the physical temperature of the $A$ ring increase as the distance to Saturn decreases.

The best model for $B(\tau)$ in the A ring (all data included) is shown in Figure 2, model $b$, along with the best zero-gradient model. The latter model yields an rms residual value slightly above the lo limit. The fit of these two models to the A ring brightness temperature as a function of $\mu$, for observations against space, is shown in Figure 3.

\section{CASSINI Division}

The Cassini division shows up as a bright narrow region between the A and B rings in Figure 1, since it is optically thinner than these rings and obstructs less planetary emission. As in the case of the A ring, there are observations over the division, both against space and against Saturn. We had to select from the raw data the points that did not appear to be significantly contaminated by the neighboring rings or the planet itself (when viewing against space). We obtained 40 observations against the planet and 20 observations against space for $1.96 \leq r / R_{s} \leq 2.00$. Both of these data sets were combined, and we now present the results of our parameter estimation, using the models described above. A temperature gradient as a function of $\tau$ does not produce a significant change in the behavior of the residuals. One would expect much less shadowing in a low optical depth region such as the Cassini division than in the A or B rings [Froidevaux, 1980], and it would not be surprising to find a much shallower gradient in this division, even for the low solar elevation angle $\left(3^{\circ}\right)$ at the time of the Pioneer Saturn flyby. We therefore proceed under the assumption that $\alpha$ is equal to zero.

If we then use (2) as our basic, two-parameter model, $\tau_{0}$ and $B_{0}$ (constants) can be determined from the (nonlinear) least squares fit to the data. Our formal results are $\tau_{0}=0.13_{-0.04}^{+0.03}$ and $T_{0}=71 \pm 5 \mathrm{~K}$. We arrived at this conclusion, after analyzing the available data in various ways, in order to investigate the effect of possible contamination from the $A$ and $B$ rings. Overall, the minimum rms residual is about 2.0 , which is slightly above the noise level in the data, suggesting that the effects of contamination are small.

\section{B RING}

The outer and inner parts of the $B$ ring are considered separately. To avoid contamination, the boundaries are taken to be from 1.65 to $1.90 R_{S}$ and from 1.55 to $1.62 R_{S}$, respectively. Over 300 observations were used for the outer $B$ ring. Eight of

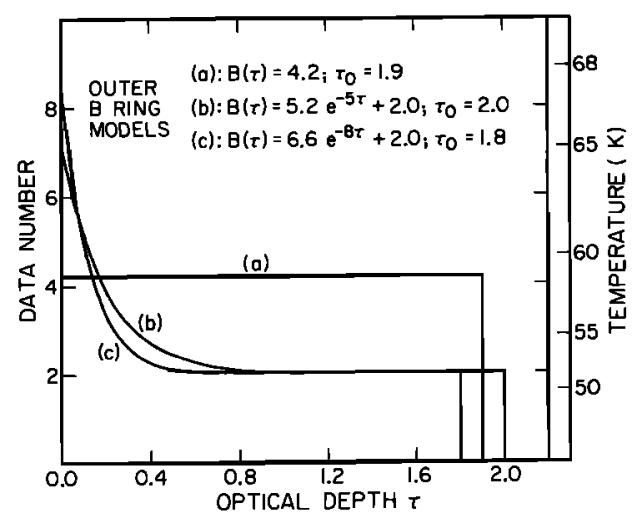

Fig. 4. Outer B ring models of the intrinsic ring brightness as a function of $\tau$. The value of $\tau_{0}$ for the constant temperature case (model $a$ ) was chosen between the values of models $b$ and $c$, because the minimum rms residual for model $a$ occurs for unreasonably high $\tau_{0}$ values $(>3)$ and is still significantly higher than $\epsilon_{\min }$. The constant brightness of 4.2 minimizes $\epsilon$ for $\tau_{0}=1.9$. For the given values of $\alpha$ and $B_{U}\left(\approx B_{2}\right)$ in models $b$ and $c$ the parameters $\tau_{0}$ and $B_{I}$ are chosen such that the rms residual is minimized. 
these are with space as a background, at elevation angles of $29^{\circ}-30^{\circ}$. The other observations are with Saturn as a background. Both data sets are needed in order to constrain the various parameters. A dozen other observations were made at very low elevation angles $\left(2^{\circ}-3^{\circ}\right)$ and were not included in the formal solution because of contamination from the $\mathbf{A}$ or $\mathbf{C}$ ring. However, these low-elevation observations can be used to set an upper limit of $71 \mathrm{~K}$ for the outer B ring brightness temperature.

For the outer $B$ ring the preferred brightness temperature $T_{I}$ of the illuminated side is $68_{-5}^{+3} \mathrm{~K}$. The value of $\tau_{0}$ is larger than 1.5 , the preferred value being between 1.7 and 1.8. The brightness temperature $T_{U}$ of the unilluminated side is not well determined, but the maximum allowed value is $56 \mathrm{~K}$. There is good evidence for a gradient within the rings, as shown in Figures 4 and 5. Model $a$ is for the two-parameter model, with the gradient parameter $\alpha=0$. The residuals, however, show a definite trend with $\mu$, as shown in Figure 5a. Positive residuals are associated with low elevation angles (low $\mu$ ), and negative residuals with high $\mu$. This trend is removed when nonzero values of $\alpha$ are introduced, as in Figures $5 b$ and 5c. The bounds on $\alpha$ (as well as $\tau_{0}$ and $T_{l}$ ) are given in Table 2.

The inner part of the B rung has a smaller optical depth than the outer part, the allowed values being in the range $0.85-1.15$ (preferred $\tau_{0}=0.95$ ). A zero gradient is just within the allowed bounds on $\alpha$, with a constant temperature of 66

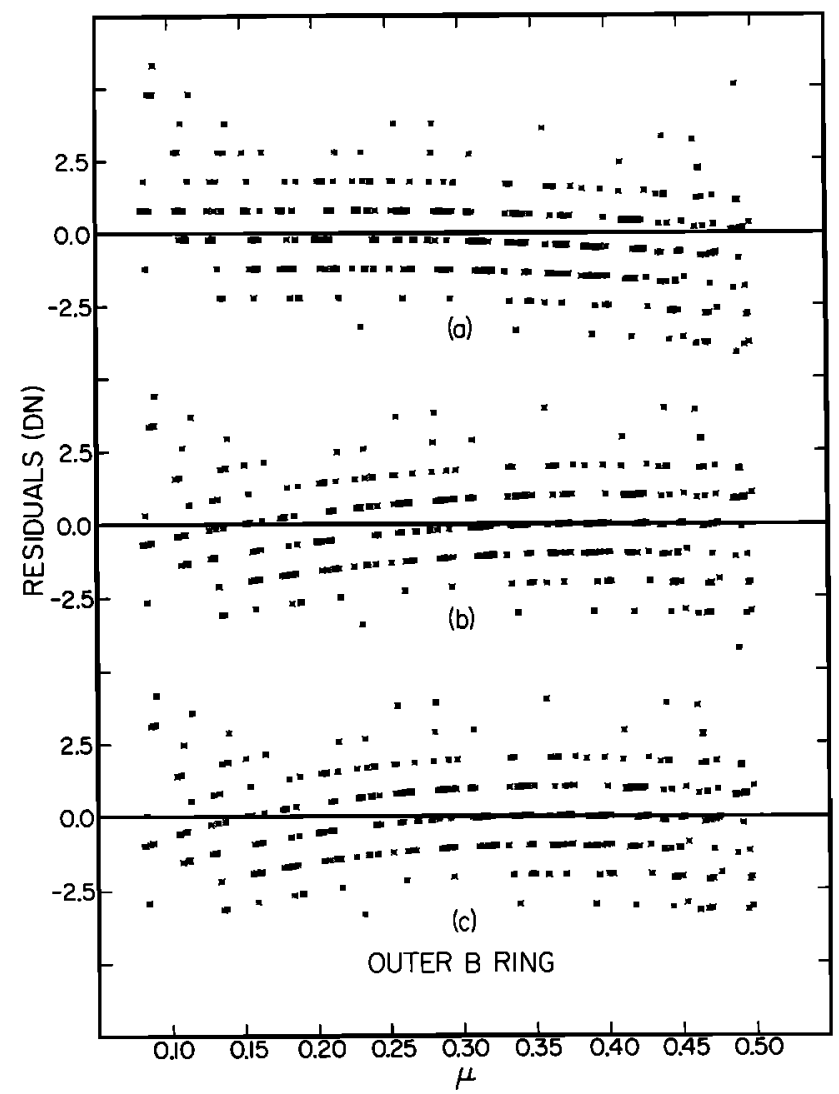

Fig. 5. Outer B ring residuals (versus $\mu$ ) for models $a, b$, and $c$ (from top to bottom) of Figure 4. If no significant temperature gradient exists within the ring (model $a$ ), the residuals are positive for small $\mu$ and negative for large $\mu$. The effect of the gradient is to center the residuals about zero and to decrease the rms residual value. The discrete values of the residuals are due to the digitization of the observed ring data.

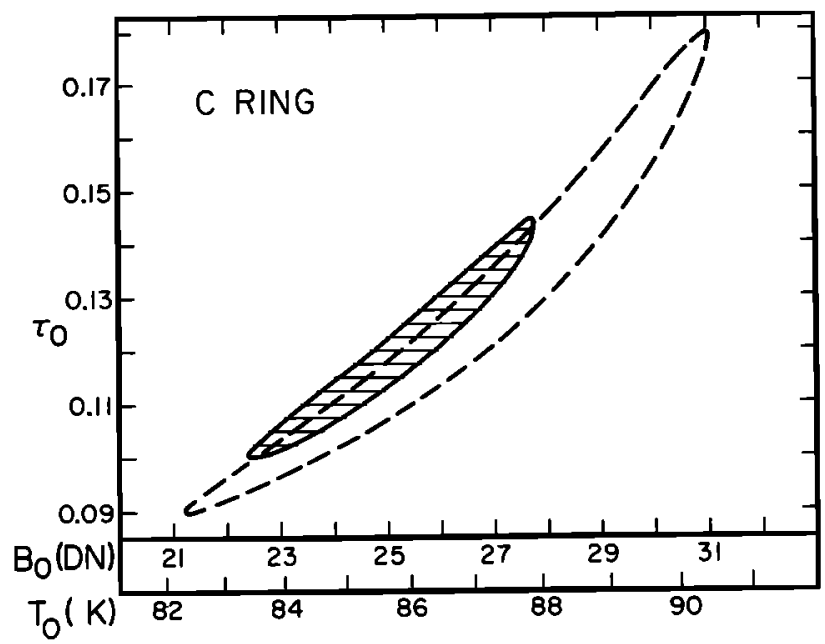

Fig. 6. Bounds on $\tau_{0}$ and $B_{0}$ for the $C$ ring. No temperature gradient within the ring is used, since it does not significantly change the residuals. The dashed curve is for a simple two-parameter $\left(\tau_{0}, B_{0}\right)$ model described by equation (2). The solid curve is for a four-parameter $\left(\tau_{0}, B_{0}\right.$, and their radial gradients) model described in the text. The region enclosed by the solid curve represents all allowable $\tau_{0}$ and $B_{0}$ values, when the radial gradients are varied within as much as one standard deviation from their preferred values. $B_{0}$ and $\tau_{0}$ now refer to the values at $1.35 R_{S}$.

K. However, positive values of $\alpha$ are preferred, with a corresponding increase in the illuminated side temperature $T_{I}$ above $66 \mathrm{~K}$. The best fit is for $T_{I}=70_{-3}^{+5} \mathrm{~K}$. The unilluminated side temperature $T_{U}$ must be less than $65 \mathrm{~K}$.

\section{RING}

The $C$ ring data set includes over 300 observations between 1.25 and $1.45 R_{S}$ (our choice of reduced boundaries) with planet as a background. The $C$ ring residuals, like those of the Cassini division, are not sensitive to the gradient parameter $\alpha$. We therefore set $\alpha=0$ and proceed with the solution, using (4). The allowed solution is enclosed by the dashed curve in the $\left(\tau_{0}, B_{0}\right)$ plane as shown in Figure 6 . The preferred values are $\tau_{0}=0.13$ and $T_{0}=87 \mathrm{~K}$ for this model.

However, the rms residual for the two-parameter model is 2.8 DN, which is significantly higher than the noise. Also, there is a systematic trend in the residuals as a function of radius, as shown in Figure 7a. Positive residuals occur close to the planet, and negative residuals occur farther away. This trend is removed by assuming a linear increase in $\tau_{0}$ with increasing $r$, as shown in Figure $7 b$, or by assuming a linear decrease in $B_{0}$ with increasing $r$. When a variation of $\tau_{0}$ is assumed, the best solution is for $\tau_{0}=0.04$ at $r=1.25 R_{S}$ and $\tau_{0}$ $=0.20$ at $r=1.45 R_{S}$, with $T_{0}$ about $86 \mathrm{~K}$. When a variation of $T_{0}$ is assumed, the best solution is for $T_{0}=94 \mathrm{~K}$ at $1.25 R_{S}$ and $T_{0}=80 \mathrm{~K}$ at $1.45 R_{S}$, with $\tau_{0}$ about 0.12 . The residuals $\epsilon$ are significantly lower (in the sense of (8)) when $\tau_{0}$ is varied than when $T_{0}$ is varied. When both parameters are varied together, the preferred solution has $T_{0}$ roughly constant at $86 \mathrm{~K}$. The minimum residual $\epsilon_{\min }$ is then about $1.85 \mathrm{DN}$, which is close to the noise of a single observation.

Allowing $\tau_{0}$ and $B_{0}$ to vary with radial distance $r$ also reduces the uncertainty in $T_{0}$ and $\tau_{0}$ (at $r=1.35 R_{S}$ ), as shown by the shaded area of Figure 6. We do not understand this preference for a model in which the temperature of the $\mathrm{C}$ ring is independent of $r$. These particles are significantly hotter than particles at greater radii, where the effect of radiation 


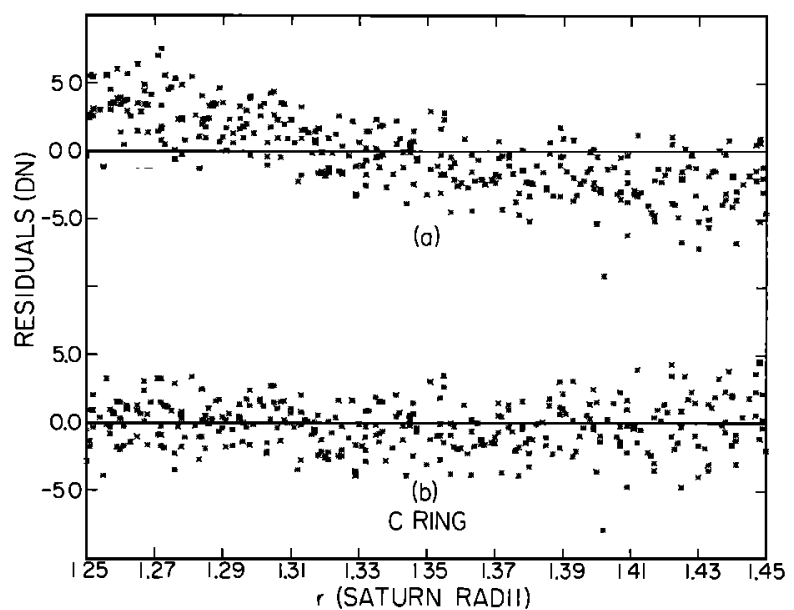

Fig. 7. $C$ ring residuals versus distance $r$ from Saturn. The upper plot (plot $a$ ) corresponds to the best two-parameter $\left(\tau_{0}, B_{0}\right)$ model, with $\tau_{0}=0.13$ and $B_{0}=27.2\left(T_{0}=87.5 \mathrm{~K}\right)$. The lower plot (plot $b$ ) results from a four-parameter model, allowing for a decrease in $\tau_{0}$ and an increase in $B_{0}$ toward the planet. The minimization of the residuals in this case yields $\tau_{0}=0.12$, and $B_{0}=25.5\left(T_{0}=86 \mathrm{~K}\right)$. The systematic variation apparent in plot $a$ has been removed in plot $b$ by allowing $\tau_{0}$ to vary between 0.04 and 0.20 in the region between 1.25 and $1.45 R_{S}$. The digitization of the data is not as apparent as in Figure 5 (outer $B$ ring), because the main contribution comes from the planetary emission, which is a continuous function (of emission angle).

from Saturn is less. However, this reasoning leads us to expect a radial temperature gradient in the $\mathrm{C}$ ring, which apparently is not necessary in our solution, when four free parameters are included.

There are exactly seven observations of the south side of the $C$ ring in the planet's shadow (time in eclipse $=5000 \pm 500 \mathrm{~s}$ ). Values of brightness temperature range from 59 to $67 \mathrm{~K}$. Because contamination with space is occurring (the observations are with space as a background at instrument elevation angles of $1^{\circ}$ ), the high values are the most significant. Also, brightness temperature is less than physical temperature for a partly transparent medium like the $\mathrm{C}$ ring. Thus the physical temperature may be $70 \mathrm{~K}$ or more. However, a $15-\mathrm{K}$ drop during eclipse is significant and implies a low value for the thermal inertia of $\mathbf{C}$ ring particles.

Adjacent to the seven observations of the $\mathrm{C}$ ring in eclipse we have seven poorly resolved observations of the $A$ ring, $2000 \mathrm{~s}$ after the end of eclipse. Brightness temperatures range from near zero to $63 \mathrm{~K}$ and are clustered between 52 and 56 $K$. Contamination from the $\mathrm{C}$ ring and space is severe, but a 10-K temperature drop is not inconsistent with earth-based observations and low thermal inertia surfaces [Froidevaux et al., 1980; G. Rieke, private communication, 1980].

\section{SUMMARY AND DISCUSSION}

The main results from our modeling of the Pioneer Saturn infrared radiometer observations (at $45 \mu \mathrm{m}$ ) are summarized in Tables 1 and 2. The various ring boundary locations are shown in Table 1, while the preferred parameter values, as determined by the location of the minimum rms residual, are listed in Table 2. Figures 8 and 9 display the physical temperature $T_{I}$ of the illuminated side of Saturn's rings and the optical depth $\tau_{0}$, respectively, as a function of radial distance from the planet. We briefly discuss these results and compare our parameter estimates with previous, earth-based estimates. We emphasize that our results are model-dependent, since we have assumed that the rings are many particles thick.
Both the observations and the parameter estimation point to a substantial temperature gradient within Saturn's rings. The existence of such a temperature gradient (at least within the A and B rings) does not rule out the possibility of a monolayer. Such a ring would also exhibit hotter temperatures on the illuminated side. However, one might expect collisions between the particles in an optically thick ring (such as the outer B ring), and the tumbling of particles in space might tend to smooth out the temperature difference that one would observe from opposite sides of the ring. Note that the particles in a monolayer of optical depth larger than 1.5 would have to be tightly squeezed together. A strong temperature gradient with $\tau$ is easily understood in terms of a thick multilayer of particles, illuminated at low elevation angle by the sun. The particles in such a ring would travel from the illuminated to the unilluminated side once per orbit, and they would have to cool within a few hours by approximately $10^{\circ}$. This is in accordance with the eclipse data, as well as with analyses of earth-based cooling and heating observations, and low thermal inertia surfaces are indicated. The monolayer versus multilayer question cannot be definitely closed. Price [1976], in his synthesis of the various ring models, had pointed out the likelihood of a temperature gradient within the rings.

The optical depth estimates for the various regions can be compared to earth-based determinations; these generally lie somewhat lower than the infrared radiometer results. Cuzzi [1978] has recently summarized the available earth-based determinations of $\tau_{0}$ for Saturn's rings. The value of $\tau_{0}$ for the average B ring (inner and outer) is probably not larger than unity, according to most previous observations. We find that a value of 1.3 or higher results from the Pioneer Saturn infrared data. Our results for the outer region agree with the conclusion of the photopolarimeter team [Gehrels et al., 1980] that $\tau_{0}$ is larger than 1.5 in a large portion of the B ring. These authors also indicate that 'in at least 5 percent of the surface area of the B ring the optical depth is less than 0.25.' Our average results for both the inner and the outer regions do not appear sensitive to this effect. The earth-based results are also modeldependent, and it is possible to underestimate $\tau_{0}$ in the infrared, for example, by omitting the brightness contribution

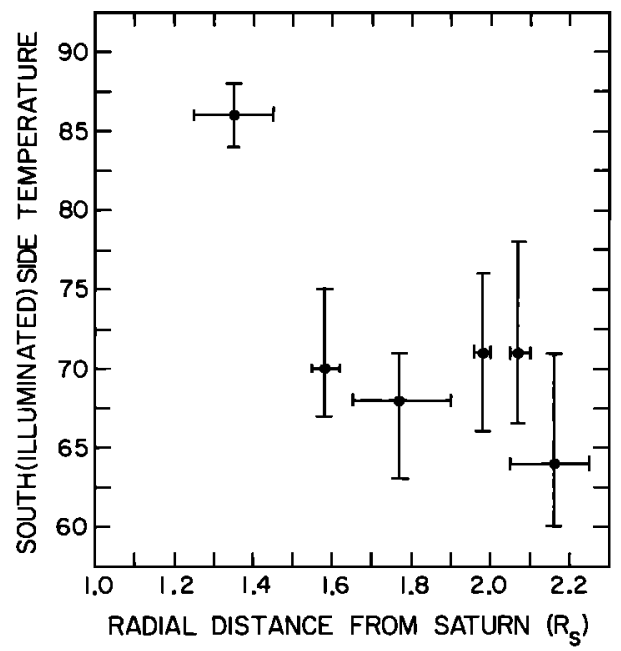

Fig. 8. Physical ring temperature on the south (illuminated) side as a function of radial distance from Saturn. The values are taken from Table 2, and the horizontal error bars indicate the radial range over which the observations leading to the parameter estimates were made. 


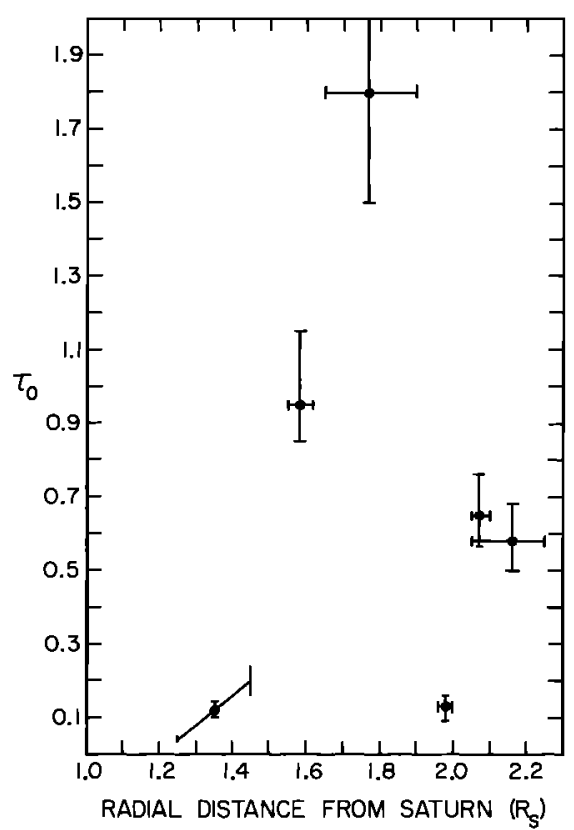

Fig. 9. Total normal optical depth as a function of radial distance from Saturn. The upper limit in the case of the outer B ring (average distance of $1.77 R_{S}$ ) is not well constrained by the data. Note the increase in optical depth from the inner to the outer edge of the $\mathrm{C}$ ring.

from the ring itself when considering transmitted radiation through the ring.

According to earth-based determinations the average A ring optical depth is most probably between 0.3 and 0.7 . Our results lean toward the higher values. The optical depths of both the $\mathrm{C}$ ring and the Cassini division are close to 0.1, according to our least squares solutions. The optical depth of the $\mathrm{C}$ ring is found to decrease toward Saturn. This is consistent with the virtual absence of a D ring [Gehrels et al., 1980]. An estimate of the C ring optical depth by Ferrin [1974], based on the eclipse observations of Iapetus by Barnard [1890], is in good agreement with our determination of a decrease in $\tau_{0}$ from 0.20 (at $1.45 R_{S}$ ) to 0.04 (at $1.25 R_{S}$ ). Ferrin [1974] also found a nonzero value of $\tau_{0}$ for the $D$ ring (about 0.02 ), but the Pioneer Saturn photopolarimeter observations [Gehrels et al., 1980] point to an order of magnitude lower value. This could indicate real temporal variations in the very sparsely populated regions of Saturn's rings. Such a possibility for the Cassini division was advocated by Hämeen-Anttila [1977], and the estimates of $\tau_{0}$ for this region vary by orders of magnitude. Whether these variations are real or a product of uncertainties in the difficult estimates is not clear. Even though Bobrov [1970] and Hämeen-Anttila [1977] point to a value between $10^{-3}$ and $10^{-4}$ for the division's optical depth, the results of Coupinot [1973], Ferrin [1975], Lumme [1975], Fountain [1977], and Lumme and Reitsema [1977] are much closer to our determination. Our result is still a factor of 2 larger than the average from the above five references.

If one considers the temperature bounds on the various regions, one notes a substantial warming as the distance from Saturn decreases. Independent modeling of the behavior of Saturn's rings as a function of solar elevation angle [Froidevaux, 1980] also indicate brightness temperatures between 60 and $65 \mathrm{~K}$ for the $\mathrm{A}$ and $\mathrm{B}$ rings for the geometry applicable to the Pioneer Saturn flyby. In the case of the $\mathrm{C}$ ring such high temperatures are understood if the particles have high (water frost type) Bond albedos and rotate slowly with respect to the sun or, alternatively, if they are isothermal, fast rotators, but with darker surfaces than the icy surfaces of the $A$ and $B$ ring particles. As far as the temperature increase between the outer and the inner edges of the $C$ ring is concerned, it is hard to believe that it is nonexistent, as seems to be implied by the analysis of the residuals, when both $\tau_{0}$ and $B_{0}$ are allowed to vary with $r$. If the particles in the $C$ ring are very dark, the solar contribution could be enhanced in relation to Saturn's effect, which would smooth out the temperature variation in this ring. While this effect might play a role, it still seems that some artifact in the data analysis is the most likely explanation for this apparent lack of temperature increase in the C ring.

The general trend abserved from Table 2 is toward an increase in temperature as $r$ decreases and Saturn's effect becomes more important. The apparently higher physical temperature in the Cassini division versus the outer $B$ ring (see Figure 8) can be understood in terms of the smaller shadowing effect in the Cassini division. That is, a particle in the optically thin Cassini division receives more sunlight than one in the optically thick B ring. It is interesting to compare the temperatures in the $\mathrm{C}$ ring with those in the Cassini division. Since these two regions have similar optical depths, the main difference between the temperatures should come from the effect of Saturn's proximity (for similar particles in both regions). Simple modeling indicates that the ratio of effective solid angles subtended by Saturn at the $C$ ring and the Cassini division is between 2 and 2.5. The data point to a ratio of emitted flux in the $\mathrm{C}$ ring to emitted flux in the Cassini division of about $2.2 \pm 1$. These numbers are in approximate agreement, since the absorbed sunlight is small $(\sim 10 \%$ for the $\mathrm{C}$ ring) in comparison to absorbed radiation from Saturn at the time of the Pioneer flyby. This conclusion depends on an assumed albedo and other model parameters that are not well known. Thus our data do not resolve the question of whether $\mathrm{C}$ ring particles are different from those in the Cassini division. More resolution in the temperature and optical depth profiles as a function of radial distance would help in understanding the trade-off between ring optical and physical thickness (which affect shadowing and heating between particles), distance from Saturn, and other parameters such as particle albedo and rotation rate, which all affect the temperature structure in Saturn's rings. The upcoming Voyager flyby of Saturn should help to elucidate some of the remaining questions, and we hope to learn more about the temperatures, optical depths, and inhomogeneities in Saturn's rings.

\section{REFERENCES}

Barnard, E. E., Observations of the eclipse of Iapetus, Mon. Not. $R$. Astron. Soc., 50, 107-110, 1890.

Bevington, P. R., Data Reduction and Error Analysis for the Physical Sciences, McGraw-Hill, New York, 1969.

Bobrov, M. S., Physical properties of Saturn's rings, in Surfaces and Interiors of Planets and Satellites, edited by A. Dollfus, pp. 376-461, Academic, New York, 1970.

Coupinot, G., Les anneaux de Saturne en 1969: Etude morphologique et photométrique, II, Déconvolution des courbes photométriques brutes, Icarus, 19, 212-223, 1973.

Cuzzi, J. N., The rings of Saturn: State of current knowledge and some suggestions for future studies, NASA Conf. Publ., 2068, 73$104,1978$.

Ferrin, I. R., Saturn's rings, I, Optical thickness of rings A, B, D and structure of ring B, Icarus, 22, 159-174, 1974.

Ferrin, I. R., Saturn's rings, II, Condensations of light and optical thickness of Cassini's division, Icarus, 26, 45-52, 1975.

Fountain, J. W., Saturn's ring: Physical thickness and the optical 
depth of Cassini's division and ring C, Bull. Am. Astron. Soc., 9, 463, 1977.

Froidevaux, L., Saturn's rings: Infrared brightness variation with solar elevation, submitted to Icarus, 1980.

Froidevaux, L., K. Matthews, and G. Neugebauer, Thermal response of Saturn's ring particles during and after eclipse, submitted to Icarus, 1980.

Gehrels, T., et al., Imaging photopolarimeter on Pioneer Saturn, Science, 207, 434-439, 1980.

Hämeen-Anttila, K. A., Optical thickness of the Cassini division, Icarus, 31, 385-400, 1977.

Ingersoll, A. P., G. S. Orton, G. Münch, G. Neugebauer, and S. C Chase, Pioneer Saturn infrared radiometer: Preliminary results, Science, 207, 439-443, 1980.

Loewenstein, R. F., D. A. Harper, Jr., H. Moseley, E. Shaya, and J. Smith, Far-infrared observations of Titan and Iapetus, Icarus, in press, 1980.

Lumme, K., Shape of the Cassini division in different colors, Icarus, 24, 483-491, 1975.
Lumme, K., and H. J. Reitsema, Five color photometry of Saturn and its rings, Bull. Am. Astron. Soc., 9, 463, 1977.

Orton, G. S., and A. P. Ingersoll, Saturn atmospheric temperature structure and heat budget, $J$. Geophys. Res., this issue.

Price, M. J., Infrared thermal models for Saturn's ring, Icanus, 27, 537-544, 1976.

Tokunaga, A. T., J. Caldwell, and I. G. Nolt, The $20 \mu \mathrm{m}$ brightness temperature of the unilluminated side of Saturn's rings, Nature, in press, 1980.

Van Allen, J. A., M. F. Thomsen, B. A. Randall, R. L. Rairden, and C. L. Grosskreutz, Saturn's magnetosphere, rings, and inner satellites, Science, 207, 415-421, 1980.

(Received May 6, 1980;

revised June 19, 1980;

accepted June 21,1980 .) 